\title{
Importance of the Pneumococcus in Community-acquired Pneumonia in Tender-age Infants on the Background of Vaccination
}

\author{
B. T. Tukbekova ${ }^{1}$, Aliya A. Zhanpeissova ${ }^{1 *}$, S. B. Akhmetova ${ }^{2}$, B. Dyussenova Sandugash ${ }^{1}$, K. Zh. Alimshaikhina ${ }^{1}$, \\ G. A. Sarmankulova ${ }^{1}$
}

${ }^{1}$ Department of Pediatrics and Neonatology, NJSC "Medical University of Karaganda," Karaganda Medical University, Karaganda City, Kazakhstan; ${ }^{2}$ Department of Biomedicine, NJSC "Medical University of Karaganda," Karaganda Medical University, Karaganda City, Kazakhstan

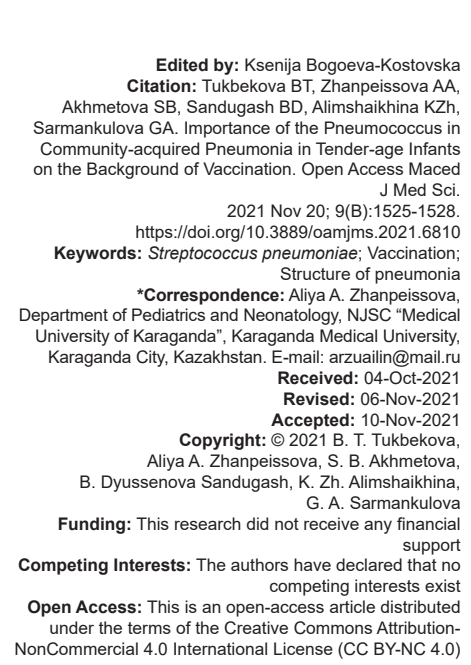

\section{Introduction}

Community-acquired pneumonia is the most common form of pneumonia and remains one of the leading causes of the death. According to the World Health Organization (WHO), the mortality rate of children in the first 5 years of life due to pneumonia is up to $20-25 \%$ [1], [2]. All this determines the significance of the problem of pneumonia in children not only for pediatrics, but also for clinical medicine in general.

According to the $\mathrm{WHO}$, about 150 million cases of pneumonia in children under 5-years-old are registered annually in the world, from which 20 million are severely requiring hospitalization [3], [4]. In developed countries, the incidence is $3.3 / 1000$ children under 5 -years-old and 1.5 when calculating the indicator per 1000 children aged 0-16 years [5].

\begin{abstract} MATERIALS AND METHODS: In this research, 82 children with community-acquired pneumonia we examined. They divided into two groups, the first group included $42(51.2 \%)$ vaccinated children with community-acquired pneumonia. analysis was carried out using the STATISTICA package. Relative frequency of occurrence of the trait in different groups p was determined by the confidence interval statistically significant differences were considered at was detected less often. The Staphylococcus family was represented with such pathogenic strains as: Staphylococcus 01) - Staphylococcus haemolyticus. Gram-negative flora was mainly represented by Haemophilus (uenzae, Mycoplasma pneumoniae, Escherichia coli, Klebsiella pneumoniae, less often Enterobacter aerogenes, and Klebsiella oxytoca. The etiological structure of CAP in young children was characterized by the predominance $(62.5 \%, p<0.001)$ of Gram-positive flora in the group of children with impaired immunization. Significant differences are
found between groups of children. Therefore, in vaccinated children, concurrent infection was prevailed $(42.9 \%)$. And in the group of infants with impaired immunization was detected Streptococcus pneumoniae (37.5\%). Aforecited indicates CONCLUSION: Thus, the applied microbiological research methods make it possible to improve the efficiency of timely diagnosis of community-acquired pneumonia in vaccinated infants with pneumococcal vaccine at the stationary level, to improve the individual prognosis of the disease course.

AlM: The aim of the study was to study the role of pneumococcus in community-acquired pneumonia in young RESULTS: Our research revealed that the etiological structure of CAP in vaccinated children is represented by pathogenic
\end{abstract}

According to the statistical data [6] in the Republic of Kazakhstan, the absolute incidence of pneumonia in children from 0 to 5 -years-old was $42,236 / 1000$ children in 2013 year (24.0), in 2014 - 40,078 (22.8). Considering these data, it should be noted that the incidence of pneumonia in children of $0-5$-years-old has been decreased by 2158 cases (1.2) compared to 2013 year, but an upward trend also prevails in Akmola (1501 case-18.1), West Kazakhstan (282 case-4.4), and North Kazakhstan (113 case-3.9) regions.

In Kazakhstan, despite the successes achieved in pediatrics, the incidence of the respiratory diseases does not tend to decrease (2016 year - 1143.3 cases, 2017 - 1468.3) [7]. Despite significant progress in the diagnosis and treatment of community-acquired pneumonia, complications and mortality are still increasing. 
Streptococcus pneumoniae remains the main causative agent of community-acquired pneumonia in all age groups [8], [9]. Pneumococcal infections - are the group of widespread anthroponotic diseases caused by $S$. pneumoniae, representing an urgent problem for practical healthcare in all countries of the world. $S$. pneumoniae is the main cause of morbidity and mortality from respiratory infection worldwide, which contributed to increasing mortality in 2016 year $-1,189,937$ cases more than all other etiologies combined [10]. In this regard, to create specific immunity, to ensure the prevention of pneumococcal infection is vaccination [11], [12]. In May 2020 year, WHO, once again, issued a warning about the importance of immunization coverage [13], considering vaccination to be the only way to significantly affect the incidence of pneumococcal disease. At the same time, since 2010 year, it has been recommended that routine vaccinations against pneumococcus be included in the national calendars of all countries.

The increase of community-acquired pneumonia mortality is due to the frequency of complications development and cases of severe inflammatory process that develops as a result of changes in the pathogenicity of microorganisms, altered premorbid immune status of the macroorganism and a number of other factors.

\section{Aim}

The aim of the study was to study the role of pneumococcus in community-acquired pneumonia in young tender-age infants on the background of vaccination.

\section{Materials and Methods}

In this research, 82 children with communityacquired pneumonia we examined. They were treated at the Regional Children's Clinical Hospital of the Karaganda city.

The age of the patients ranged from 2 months up to 3 years. A comprehensive examination of the patients was carried out and included a detailed collection of anamnesis, objective examination, laboratory studies, and methods of instrumental diagnostics. The patients were divided into two groups, the first group included $42(51.2 \%)$ vaccinated children with community-acquired pneumonia. The second one included 40 (48.8\%) children with impaired immunization to community-acquired pneumonia.

\section{Clinical and laboratory research}

The diagnosis and severity of the disease were established according to the recommendations of the
WHO for the diagnosis and treatment of communityacquired pneumonia. They were taken as the base for the national clinical protocols of the Republic of Kazakhstan [14]. The exclusion criterion was the presence of concomitant lung diseases.

To establish the etiology of community-acquired pneumonia in all patients underwent sputum analysis for microflora using microscopic and bacteriological methods before antibiotic therapy. Informed consent was obtained from the parents of each patient. Clinical and laboratory studies were carried out on the basis of the Regional Children's Clinical Hospital of the Karaganda city and at the research center of the Karaganda Medical University.

\section{Statistical analysis}

Calculations and evaluation of the obtained results were carried out on an IBM-compatible computer with the operating system Windows XP using the MS Excel 2017 software package (Microsoft), SPSS 12.0.2 and Statistica 20. Statistical analysis was carried out using the STATISTICA package. Relative frequency of occurrence of the trait in different groups (proportion) $p$ was determined by the confidence interval statistically significant differences were considered at $p<0.05$.

\section{Ethical issue}

The study was approved by the ethics committee of Karaganda Medical University and was conducted in accordance with the Declaration of Helsinki. Information received from all legal representatives of sick children for research. While sick children are in the pulmonary department, they are all standard therapy, corresponding to the diseases.

\section{Results and Discussion}

To establish etiology of the CAP in all children was underwent a microbiological study before antibiotic therapy. Sputum was collected in compliance with standard requirements and used as material for microbiological examination. A total of 82 children were examined with an established diagnosis of community-acquired pneumonia of various localization. Of these, $42(51.2 \%)$ vaccinated children and $40(48.8 \%)$ children with impaired immunization at the age from 2 months to 3 years old.

The results of isolated strains of microorganisms from the sputum of vaccinated infants from 2 months to 1 year old with severe pneumonia (Table 1) indicate the prevalence of mixed infections: Haemophilus influenza + Staphylococcus aureus - $16.7 \%(95 \% \mathrm{Cl}$ 0; $60.01),(p<0.032)$, and also, Klebsiella pneumoniae + Streptococcus beta hemolytic Group B - $16.7 \%(95 \% \mathrm{Cl}$ 
Table 1: The structure of the causative agents of community-acquired pneumonia in infants from 2 months up to 3 years old

\begin{tabular}{|c|c|c|c|c|c|c|c|}
\hline \multirow[t]{2}{*}{ Etiologic agent } & \multicolumn{3}{|c|}{$\begin{array}{l}\text { Vaccinated infants against pneumococcal infection. } \\
n=42\end{array}$} & \multicolumn{3}{|c|}{$\begin{array}{l}\text { Infants with violation of the terms of immunization } \\
\text { against pneumococcal infection. } n=40\end{array}$} & \multirow[t]{2}{*}{$\mathrm{p}$} \\
\hline & Absolute number & Relative share (\%) & $95 \% \mathrm{Cl}$ & Absolute number & Relative share (\%) & $95 \% \mathrm{Cl}$ & \\
\hline Gram-positive bacteria & 12 & 28.57 & $(0 ; 67.04)$ & 25 & $62.5 \%$ & $(30.4 ; 89.6)$ & 0.029 \\
\hline Streptococcus & 8 & $19.04 \%$ & $(0 ; 60.01)$ & 18 & $45 \%$ & $(0 ; 72.73)$ & 0.031 \\
\hline S. pneumoniae & 3 & $7.14 \%$ & $(0 ; 52.81)$ & 15 & $37.5 \%$ & $(0 ; 72.73)$ & 0.027 \\
\hline Streptococcus beta-hemolytic Group B & 2 & $4.76 \%$ & $(0 ; 52.81)$ & 0 & 0 & & 0.145 \\
\hline Streptococcus pyogenes & 3 & $7.14 \%$ & & 3 & $7.5 \%$ & & \\
\hline Staphylococcus & 4 & $9.5 \%$ & $(0 ; 52.81)$ & 7 & $17.5 \%$ & $(0 ; 52.63)$ & 0.360 \\
\hline S. aureus & 4 & $9.5 \%$ & $(0 ; 52.81)$ & 5 & $12.5 \%$ & $(0 ; 58.15)$ & 0.595 \\
\hline Staphylococcus haemolyticus & 0 & 0 & & 2 & $5 \%$ & $(0 ; 54.4)$ & 0.144 \\
\hline Gram-negative bacteria & 12 & 28.55 & $(0 ; 67.04)$ & 12 & $30 \%$ & $(0 ; 72.73)$ & 0.560 \\
\hline Klebsiella oxytoca & 2 & $4.76 \%$ & $(0 ; 52.81)$ & 0 & 0 & & 0.145 \\
\hline Enterobacter aerogenes & 0 & 0 & & 2 & $5 \%$ & $(0 ; 54.4)$ & 0.144 \\
\hline K. pneumoniae & 2 & $4.76 \%$ & $(0 ; 56.44)$ & 1 & $2.5 \%$ & & 0.069 \\
\hline H. influenzae & 4 & $9.5 \%$ & & 2 & $5 \%$ & & \\
\hline Mycoplasma pneumoniae & 3 & $7.14 \%$ & & 3 & $7.5 \%$ & & \\
\hline Escherichia coli & 1 & $4.76 \%$ & $(0 ; 49.17)$ & 3 & $7.5 \%$ & $(0 ; 58.15)$ & 0.139 \\
\hline$P$. aeruginosa & 0 & 0 & & 1 & $2.5 \%$ & $(0 ; 50.62)$ & 0.311 \\
\hline Concurrent infection & 18 & $42.9 \%$ & $(8.45 ; 77.27)$ & 3 & $7.5 \%$ & $(0 ; 50.62)$ & 0.003 \\
\hline Combined infection: $S$. pneumoniae $+P$. aeruginosa & 2 & $4.76 \%$ & $(0 ; 49.17)$ & 1 & $2.5 \%$ & $(0 ; 50.62)$ & 0.972 \\
\hline Streptococcus beta-hemolytic Group B $+P$. aeruginosa & 3 & $7.14 \%$ & $(0 ; 52.81)$ & 1 & $2.5 \%$ & & 0.145 \\
\hline H. influenza+S. aureus & 7 & $16.7 \%$ & $(0 ; 60.01)$ & 0 & 0 & & 0.032 \\
\hline K. pneumoniae + Streptococcus beta hemolytic Group B & 6 & $14.3 \%$ & $(0 ; 52.81)$ & 1 & $2.5 \%$ & & 0.145 \\
\hline
\end{tabular}

0; 60.01), and Streptococcus beta-hemolytic Group B + Pseudomonas aeruginosa - 7.14\% (95\% Cl 0; 52.81). Gram-positive bacteria: Streptococcus beta-hemolytic Group B - 4.76\% (95\% Cl 0; 52.81), S. aureus - 9.5\% (95\% $\mathrm{Cl} 0 ; 52.81)$. In children of this group, the detection rate of S. pneumoniae $-7.14 \%(95 \% \mathrm{Cl} 0 ; 52.81)$ did not differ from the indicators of this group. Gram-negative bacteria were also presented in the form of $H$. influenzae $-9.5 \%$ (95\% Cl 0; 52.81), Mycoplasma pneumoniae - $7.14 \%$ (95\% Cl 0; 60.01), and Klebsiella oxytoca and Escherichia coli-4.76\% (95\% Cl 0; 49.17). In this group, mixed infection with $S$. pneumoniae $+P$. aeruginosa was detected in $4.76 \%(95 \% \mathrm{Cl} 0 ; 49.17)$ of all cases.

S. pneumoniae took the leading place in the microbiological picture in infants with a violation of the terms of immunization against pneumococcal infection - 37.5\% (95\% Cl 0; 72.73) ( $p<0.037)$. Among the representatives of Gram-positive flora, $S$. aureus prevailed $-12.5 \%(95 \% \mathrm{Cl} 0 ; 52.81)$ and Streptococcus pyogenes - $7.5 \%(95 \% \mathrm{Cl} 0 ; 54.4)$. In the results of studies of this group, Gram-negative flora was represented by Enterobacter aerogenes - 5\% (95\%

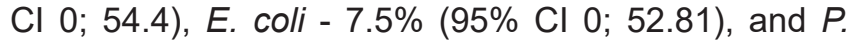
aeruginosa - 2.5\% (95\% Cl 0; 50.62). Mixed infection with S. pneumoniae + $P$. aeruginosa and $K$. pneumoniae + Streptococcus beta hemolytic Group B occurred in $2.5 \%(9 \% \mathrm{Cl} 0 ; 50.62)$ of cases.

Thus, in the group of vaccinated infants, an etiologically significant pathogen was identified in $42.9 \%$ (95\% Cl 8.45; 77.27) ( $p<0.003)$. They were represented by mixed infection $H$. influenzae $+S$. aureus $-16.7 \%$ (95\% Cl 0; 60.01) ( $<<0.032)$, while S. pneumoniae prevailed in children with impaired immunization $-37.5 \%$ (95\% Cl 0; 72.73) $(p<0.037)$ (Table 1).

The study of the microbiological structure indicates the predominance of $62.5 \%(p<0.05)$ of gram-positive flora in children with community-acquired pneumonia with impaired immunization $(p<0.05)$ (Figure 1). In the group of vaccinated children, concurrent infection was found in $42.8 \%$ ( $p<0.05)$.

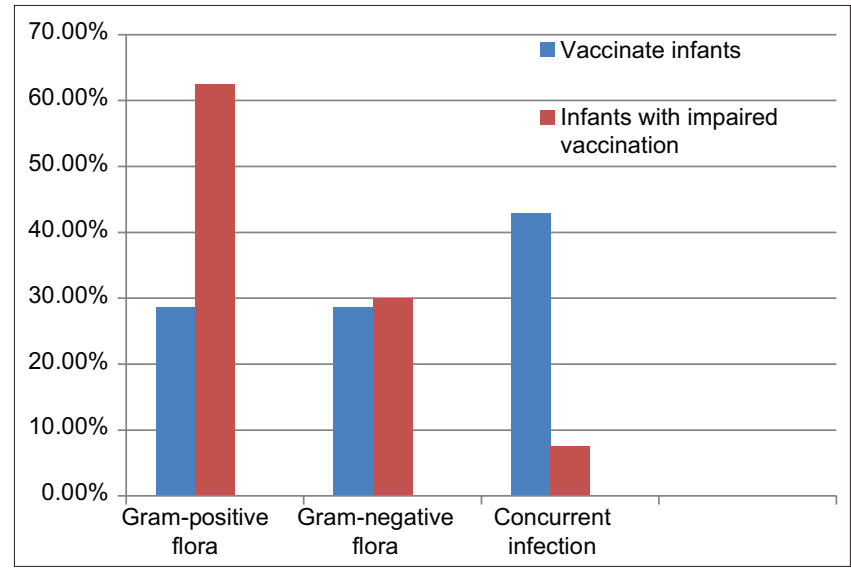

Figure 1: Bacterial flora of community-acquired pneumonia in the examined children

In case of CAP in children with impaired immunization, the majority of gram-positive microflora was represented by S. pneumoniae - 37.5\% ( $p<0.05)$. Our analysis confirms the existing information that pneumococcus is the most frequently isolated causative agent of community-acquired pneumonia in children (Figure 2).

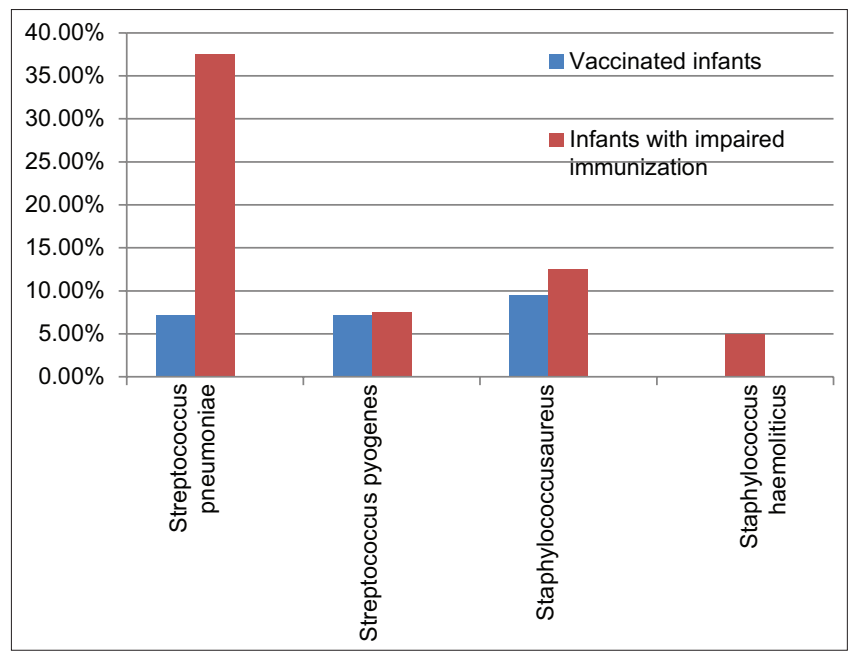

Figure 2: Gram-positive bacterial flora of community-acquired pneumonia in the examined infants 


\section{Conclusion}

Our research revealed that the etiological structure of CAP in vaccinated children is represented by pathogenic strains of Streptococci, S. pyogenes were more often detected and Streptococcus beta-hemolytic Group B were detected less often. The Staphylococcus family was represented with such pathogenic strains as: S. aureus, and less 01) - Staphylococcus haemolyticus. Gram-negative flora was mainly represented by $H$. influenzae e, M. pneumoniae, $E$. coli, K. pneumoniae, less often $E$. aerogenes, and $K$. oxytoca. The etiological structure of CAP in young children was characterized by the predominance $(62.5 \%, p<0.001)$ of Gram-positive flora in the group of children with impaired immunization. Gained results are consistent with the data of Ploskireva et al. [15], where $H$. influenzae and $S$. pneumoniae are the most significant pathogens both in monoinfections in children and in the development of concurrent infections.

Similar results were obtained by Rohde et al. [16], when this pathogen dominated the etiology of CAP, regardless of the disease severity. In the group of infants with severe pneumonia on the background of impaired immunization, $S$. pneumoniae was detected in $37.5 \%$ of cases, which proves the effect of vaccination on the etiological structure of community-acquired pneumonia.

Significant differences are found between groups of children. Therefore, in vaccinated children, concurrent infection was prevailed (42.9\%). And in the group of infants with impaired immunization was detected S. pneumoniae (37.5\%). Aforecited indicates the effect of pneumococcal vaccination on the etiological structure of CAP in tender-age infants.

Thus, the applied microbiological research methods make it possible to improve the efficiency of timely diagnosis of community-acquired pneumonia in vaccinated infants with pneumococcal vaccine at the stationary level, to improve the individual prognosis of the disease course.

\section{References}

1. 2015 Zhylda Kazakhstan Respublikasy Khalkynyk Densaulyky Zhane Densaulyk E40 Saқtau Kyymdarynyk Kyzmeti. Health of the Population of the Republic of Kazakhstan and the Activities of Health Care Organizations in 2015. Astana: Statistics Zhinaқ; 2016. p. 235

2. Begaidarova RK. Epidemiological substantiation of vaccine prophylaxis of pneumococcal infection in the Republic of Kazakhstan (Communication2). Med Ecol. 2011;1:40-4.

3. Lyutina El. Morbidity and serum from community-acquired pneumonia in children and adolescents living in Kuzbass. Pediatrician 2015;8:203-6.

4. Rudan I. Epidemiology and etiology of childhood pneumonia Bull World Health Organ 2008;86(5):408-16. http://doi. org/10.2471/blt.07.048769

PMid: 18545744

5. World Health Organization. Estimates of Disease Burden and Cost-effectiveness. Available form: http://www.who. int/immunization_monitoring/data/en. [Last accessed on 2021 May 24].

6. Shirinova M, Kulbalieva ZZ, Doltaeva BZ. Estimation of the incidence of pneumonia in children under 5 years of age in the Republic of Kazakhstan. Health and Medicine. 2017;2017:16.

7. 2017 Zhylda Kazakhstan Republics Khalkynyk Densaulyky Zhune Densaulyk E40 Sahtau Kyymdarynyk Kyzmeti. Health of the Population of the Republic of Kazakhstan and the Activities of Health Care Organizations in 2017. Astana: Statistics Zhinaқ; 2018. p. 235.

8. Levels and Trends in Child Mortality. Report 2017. Estimates developed by the UN Inter-agency Group for Child Mortality Estimation. New York: United Nations Children's Fund, World Health Organization, World Bank and United Nations; 2017.

9. Bektur CR, Nurgozhin TS. Evaluation of health outcomes and cost-effectiveness of 13-valent Pneumococcal Conjugate Vaccination for infants in Kazakhstan. Clin Ther. 2015;37(8):106.

10. GBD 2016 Lower Respiratory Infections Collaborators. Estimates of the global, regional, and national morbidity, mortality, and aetiologies of lower respiratory infections in 195 countries, 1990-2016: A systematic analysis for the global burden of disease study 2016. Lancet Infect Dis. 2018;3099(18):30310-4. http://doi.org/10.1016/S1473-3099(18)30310-4 PMid:30243584

11. Code of the Republic of Kazakhstan "On people's health and health care system" dated. Kazakhstan: Republic of Kazakhstan; 2020. p. 360.

12. Perova AL, Ruleva AA. Vaccination against pneumococcal infection. Treat Prev. 2013;4(8):43-53.

13. Least 80 Million Children Under One at Risk of Diseases Such as Diphtheria, Measles and Polio as COVID-19 Disrupts Routine Vaccination Efforts, Warn Gavi. Geneva: World Health Organization and UNICEF; 2020.

14. Pneumonia in Children. Clinical Protocols of the Ministry of Health of the Republic of Kazakhstan, Protocol No. 29. Kazakhstan Ministry of Health of the Republic of Kazakhstan; 2017.

15. Ploskireva AA, Khlypovka YN, Yatsyshina SB, Nikolaeva SV, Gorelov AV. Etiology of community-acquired pneumonia in children. RMJ Med Rev. 2018;8(2):50-4.

16. Rohde G. The Role of Viruses in CAP. European Respiratory Monograph 63: Community-acquired Pneumonia. United States: The Role of Viruses in CAP; 2014. p. 74-87. 